HIFAN 1609

Optical faraday cup for heavy ion beams

F. M. Bieniosek, S. Eylon, P.K. Roy, S.S. Yu

Accelerator Fusion Research Division

Ernest Orlando Lawrence Berkeley National Laboratory

University of California

Berkeley, California 94720

May 2005

This work was supported by the Director, Office of Science, Office of Fusion Energy Sciences, of the U.S. Department of Energy under Contract No. DE-AC02-05CH11231. 


\title{
OPTICAL FARADAY CUP FOR HEAVY ION BEAMS*
}

\author{
F. M. Bieniosek ${ }^{\#}$, S. Eylon, P.K. Roy, S.S. Yu, LBNL, Berkeley, CA 94720, USA
}

\section{Abstract}

We have been using alumina scintillators for imaging beams in heavy-ion beam fusion experiments in 2 to 4 transverse dimensions [1]. The scintillator has a limited lifetime under bombardment by the heavy ion beams. As a possible replacement for the scintillator, we are studying the technique of imaging the beam on a gas cloud. A gas cloud for imaging the beam may be created on a solid hole plate placed in the path of the beam, or by a localized gas jet. It is possible to image the beam using certain fastquenching optical lines that closely follow beam current density and are independent of gas density. We describe this technique and show preliminary experimental data. This approach has promise to be a new fast beam current diagnostic on a nanosecond time scale.

\section{INTRODUCTION}

The use of optical diagnostic techniques has become widespread in the Heavy Ion Fusion Virtual National Laboratory (HIF-VNL) [1]. However limitations to the scintillator lifetime, especially at very high beam intensity, combined with increased beam intensities by several orders of magnitude by transverse and longitudinal beam pulse compression lead to the need for a more robust technique of imaging the beam. Beam parameters range from $50 \mathrm{keV}$ to $2 \mathrm{MeV}$, with current densities from $<1$ to $>100 \mathrm{~mA} / \mathrm{cm}^{2}$, pulse lengths of 0.3 to $20 \mu \mathrm{s}$, and pulse repetition rates of 0.1 to $5 \mathrm{~Hz}$. The ideal detector in the case of high intensity beam is a material that can be replenished between successive beam pulses, such as a gas target. The gas may be generated either as a localized gas puff or gas jet introduced in the path of the beam, or as a gas cloud which is released by the beam from a solid target placed in its path. Gas molecules in the cloud excited by the beam emit visible radiation. If the gas has sufficiently high density, the radiation intensity can be shown to be independent of the local gas density, and proportional to the beam current density. As a result the optical emission intensity is a measure of the local beam current density, with properties similar to that of a Faraday cup, without the requirements for complicated electrostatic shielding. This property is especially important in the case of neutralized drift compression, in which the beam passes through a plasma, which must be carefully shielded from entering the

\footnotetext{
* Work performed under the auspices of the U.S. Department of Energy by the University of California, Lawrence Berkeley National Laboratory under Contract No. DE-AC03-76F00098. "fmbieniosek@lbl.gov
}

collection region of the Faraday cup. Here we describe the concept of the optical Faraday cup and we present initial data using the NDCX K' beam. The concept is not limited to use in heavy ion beams. It is adapted from studies of electron-induced fluorescence at near-UV wavelengths from intense electron beam pulses transported through high-density gases such as $\mathrm{N}_{2}$ and air [2-4].

\section{OPTICAL FARADAY CUP}

For the sake of illustration, we follow a particular emission line of molecular nitrogen at $391.4 \mathrm{~nm}$. This line is part of the first negative band of $\mathrm{N}_{2}{ }^{+}[5,6]$ and is studied in detail in Refs. 2-4. To model emission of the line, the history of $\mathrm{N}_{2}{ }^{+}$must be followed in detail. The optical emission intensity I per unit length for a given line with radiative transition rate $\mathrm{A}$ due to an intense ion beam intercepting a high-density gas cloud is proportional to the density of the excited state, $\mathrm{N}^{*}$ :

$$
I=h v A\left(\pi a_{b}^{2}\right) N^{*}
$$

where $\mathrm{h} v$ is the photon energy, and $\pi a_{b}^{2}$ is the cross section of the beam.

Excited states are created by direct collisions of the beam ions with the gas molecules. The time history of the excited state follows the rate equation

$$
\frac{d N^{*}}{d t}=\left(\frac{J}{e}\right) n_{g} S^{*}-\left(A+B n_{g}\right) N^{*}
$$

The first term on the right is the source term; $\mathrm{J}$ is the beam current density and $\mathrm{n}_{\mathrm{g}}$ is the local gas density. $\mathrm{S}^{*}$ is an effective cross section that represents the rate of excitation by the beam. The second term is the loss term, where $\mathrm{B}$ is the quenching rate of the line by collisional deexcitation. Performing a Fourier transform by replacing the time derivative $\mathrm{d} / \mathrm{dt}$ by $\mathrm{j} \omega$, the equation may be rewritten as

$$
N^{*}=\frac{J S^{*}}{e B\left(1+\frac{A+j \omega}{n_{g} B}\right)}
$$

When the gas density is high enough that $n_{g} B>A$, the density of excited states, and hence the optical emission, 
is independent of gas density $\mathrm{n}_{\mathrm{g}}$, and linearly dependent on the beam current density $\mathrm{J}$, so that approximately:

$$
N^{*} \cong \frac{J S^{*}}{e B} \quad\left(n_{g} B>>A\right)
$$

Specifically for the $391.4 \mathrm{~nm}$ nitrogen line, $\mathrm{A}=1.52 \times 10^{7}$ sec- 1 , and $B=4.53 \times 10^{-10} \mathrm{~cm}^{3} / \mathrm{sec}$. For the inequality to hold true, the gas density must be $n_{g} \gg n_{g 0}=A / B=3.35$ $\mathrm{x} 10^{16} \mathrm{~cm}^{-3}$, equivalent to a room-temperature pressure, $\mathrm{P}$, of about 1 Torr.

At high gas density $\left(\mathrm{n}_{\mathrm{g}}>>\mathrm{n}_{\mathrm{g} 0}\right)$, the frequency response of the diagnostic is characterized by the $3-\mathrm{db}$ frequency of eq. $3, \omega_{3 d b}=B n_{g}$. For example if the desired $\omega_{3 d b}$ is $10^{9} \mathrm{sec}^{-1}$, the requirement on $\mathrm{n}_{\mathrm{g}}$ is $\mathrm{n}_{\mathrm{g}}=2.2 \times 10^{18} \mathrm{~cm}^{-3},(\mathrm{P}$ $\approx 60$ Torr). This gas density provides time resolution in the nsec range.

Gas-jet densities much higher than this level may be readily obtained in the laboratory; for example in Ref. 7 a supersonic gas jet of density $4.5 \times 10^{19} \mathrm{~cm}^{-3}$ over distances of several $\mathrm{mm}$ was developed and used as a medium for laser-based acceleration of electron beam pulses.

In lieu of intense gas jets such as used in the work of Ref. 7, we have developed a simple passive way of generating relatively high-density localized gas clouds, using a metal wall or hole plate. Large quantities of gas are released when an ion beam is intercepted by a wall. The ratio $\mathrm{N}_{\mathrm{m}}$ of gas molecules produced to beam ions at normal incidence for $1-\mathrm{MeV}$ heavy-ion beams is approximately $\mathrm{N}_{\mathrm{m}}=10^{3}$ to $10^{4}$ [8], and the ratio is significantly higher for higher energy beams. The average gas density near the surface of the wall may be estimated by

$$
n_{g}=N_{m} \frac{J}{e} \frac{4}{\bar{v}}
$$

where $\bar{v} / 4$ is the average directed velocity of the gas cloud. (Note that the beam pulse length does not appear in this equation.) If one uses an estimate for $\mathrm{N}_{\mathrm{m}}$ of 3000 , and $\bar{v} / 4$ of $500 \mathrm{~m} / \mathrm{sec}$, the beam current density at which $\mathrm{n}_{\mathrm{g}}=\mathrm{n}_{\mathrm{g} 0}$ is $90 \mathrm{~mA} / \mathrm{cm}^{2}$. For comparison, in experiments at the HIF-VNL the beam current density in the HCX experiment is $\mathrm{j} \approx 60 \mathrm{~mA} / \mathrm{cm}^{2}$, and in the focal spot of the (uncompressed) NTX experiment the beam current density was $\mathrm{j} \approx 300 \mathrm{~mA} / \mathrm{cm}^{2}$. Planned longitudinal beam compression experiments are expected to increase the peak current density to $\mathrm{j}>10 \mathrm{~A} / \mathrm{cm}^{2}$ in a $1-\mathrm{ns}$ pulse on target. These current densities are high enough that the inequality $\mathrm{n}_{\mathrm{g}} \gg \mathrm{n}_{\mathrm{g} 0}$ is true, and the 3-db frequency $\omega_{3 d b}=B n_{g}=1.7 \times 10^{9} \mathrm{sec}^{-1}$ for a current density of $10 \mathrm{~A} / \mathrm{cm}^{2}$, which is fast enough for sub-ns response during the peak current pulse.
The desired gas density depends on the species of gas emitted by the wall, which is not well known at the present. The level of current required to obtain the desired gas density also depends on the transition and collisional de-excitation rates of the particular line to be observed.

\section{INITIAL EXPERIMENTAL TESTS}

Most initial experimental tests of the optical Faraday cup have been performed without optical filtering, including in the data presented below. Therefore optical emissions from processes which may have widely differing transition rate constants are included in these measurements. Figure 1 shows the results of a comparison of measured light emission from a scintillator, which follows closely the incident beam current density, with the light emission from a (Buckbee-Mears) hole plate. The scintillator and hole plate were each placed in the path of a tightly-focused ion beam and imaged from downstream (imaging the gas cloud on the front side of the hole plate through the holes) by a Roper Scientific intensified gated CCD camera. As the beam strikes the hole plate, a gas cloud is generated on the front surface of the plate, and the optical emission is due to excitation of the gas by the incident beam pulse. The measured optical distributions are similar but not identical in the two images, suggesting that in this case the beam current density, and thus the gas density on the surface of the hole plate, was lower than required for the inequality (3) above to hold true across the beam profile.
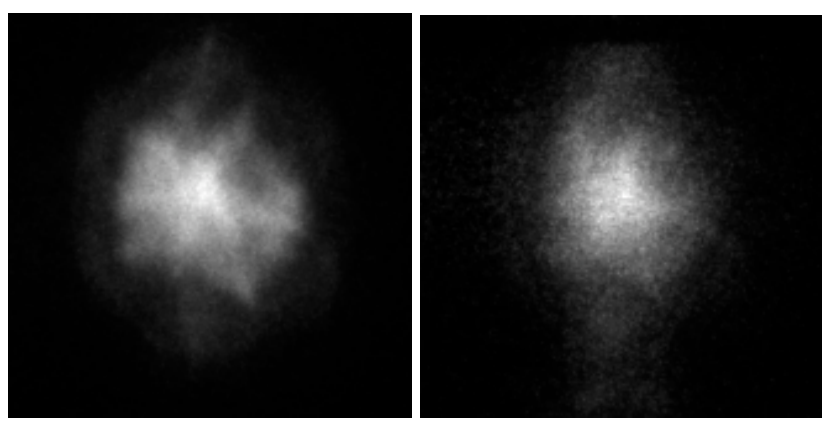

Figure 1. Comparison between beam image on scintillator (left) with beam image on gas cloud formed at hole plate (right). Scale of images is $2.5 \times 2.5 \mathrm{~cm}$. The scintillator emission is about $3 x$ higher in intensity than the emission from the hole plate gas cloud.

The time behavior of the optical emission of the gas cloud was investigated using a hole plate and a fast Hamamatsu phototube. Figure 2 shows the signal from the gas cloud as a function of time, together with the accelerating Marx voltage pulse waveform for a typical uncompressed NDCX beam [9]. Note that the voltage pulse and current pulse have a flat top, which is reflected in the optical emission signal. In addition the optical signal has a 
slowly-varying emission component which is evident in a ramped signal during the beam pulse and a long tail in the optical signal after the end of the beam pulse when the accelerating voltage is small. The slow optical component is undesired when using the gas cloud emission as a beam diagnostic and should be removed by optical filtering if possible.

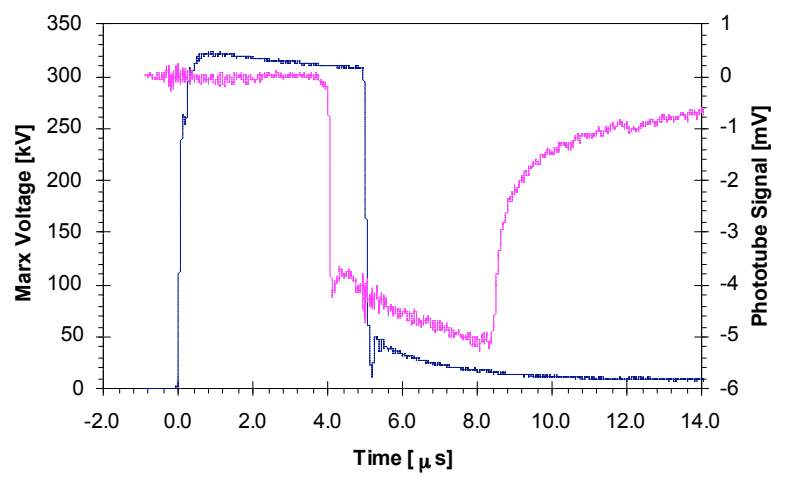

Figure 2. Marx voltage pulse (blue) and phototube signal (magenta) for a typical uncompressed NDCX beam pulse.

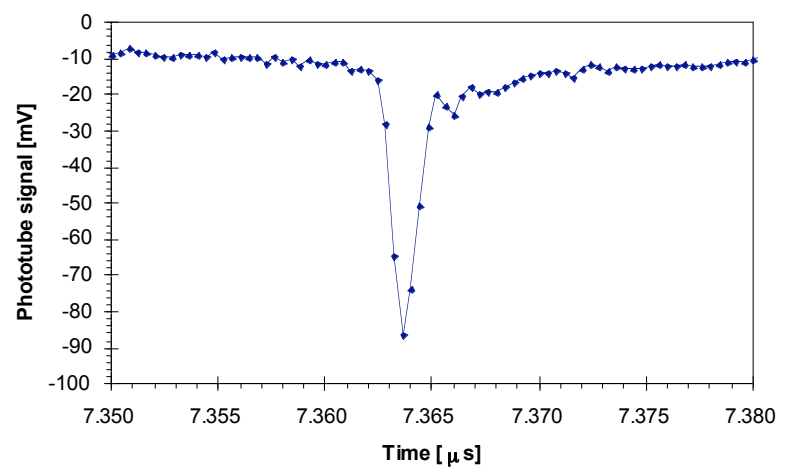

Figure 3. Fast time response of the optical emission from hole plate (phototube signal) with a compressed beam pulse. The oscilloscope sampling rate is $400 \mathrm{ps} / \mathrm{sample}$.
Measurements of the fast time response of the optical emission from the gas cloud are presented in Figure 3. In this experiment a portion of the same beam as in Figure 2 is compressed longitudinally by a factor of about 50x to produce a short intense spike in beam current. The FWHM of the spike is about $3 \mathrm{~ns}$ as measured optically using a scintillator. The beam current spike is qualitatively reproduced in the fast spike in the optical signal from the gas cloud. The speed suggests at least one optical line is fast enough to follow a compressed beam pulse with rise time on the order of 1 ns. Further investigations will utilize optical filters and spectral analysis to determine the spectral lines of greatest interest.

\section{REFERENCES}

[1] F.M. Bieniosek, L. Prost, W. Ghiorso, Proceedings PAC 2003, Portland, OR, p. 2524.

[2] D.J. Eckstrom, J.S. Dickinson, Diagnostics development for e-beam excited air channels: Optical studies of the PHERMEX beam, SRI International Corp. Report 03/1984.

[3] A.M. Frank, S.S. Yu, J.A. Masamitsu, LLNL Report UCRL-86571 (1981).

[4] Y.P. Chong, S.S. Yu, T.J. Fessenden, J.A. Masamitsu, A.M. Frank, D.S. Prono, J. Appl. Phys 55, 611 (1984).

[5] G. Davidson and R O’Neill, J. Chem. Phys. 53 (1970) 1795.

[6] R.H. Hughes, J.L. Philpot, C.Y. Fan, Phys. Rev. 123 (1961) 2084.

[7] C.G.R. Geddes, Cs. Toth, J. van Tilborg, E.Esarey, C.B. Schroeder, D. Bruhwiler, C. Nieter, and W.P. Leemans, Nature 431 (2004) 538.

[8] A.W. Molvik, M.K. Covo, F.M. Bieniosek, L. Prost, P.A. Seidl, D. Baca, A. Coorey, Phys. Rev. Spec. Topics-AB 7, 093202 (2004).

[9] P.K. Roy, et. al., Initial results on neutralized drift compression experiments (NDCX) for high intensity beam, Paper FPAE071, these proceedings. 